\title{
Treatment Uptake of Patients with Chronic Hepatitis C: Can We Expect and Do More?
}

\author{
Chia-Yen Dai
}

Published online: 26 October 2010

(C) Springer Science+Business Media, LLC 2010

Hepatitis C virus (HCV) infection frequently causes chronic liver disease leading to cirrhosis and hepatocellular carcinoma (HCC), and HCV-related end-stage liver disease is the main indicator of the need for liver transplantation in Western countries and the leading cause of death amongst cirrhotic patients $[1,2]$. To improve the overall outcome and prevent patients from the major long-term complications of chronic hepatitis $\mathrm{C}$ ( $\mathrm{CHC}$ ), we have to explore more effective antiviral therapies and more aggressive policies to encourage patients to receive therapies, since in the absence of the effective vaccine for hepatitis $\mathrm{C}$ in contrast to hepatitis $\mathrm{B}$, the standard of care (SOC) for antiviral treatment of $\mathrm{CHC}$ is combination therapy with pegylated IFN alfa (PegIFN) and ribavirin.

The optimal treatment duration and ribavirin dose were investigated in an international multicenter randomized clinical trial [3] and the standard treatment recommendations developed currently according to the HCV genotypes are quite simple to follow. Patients with genotype 1 or 4 are treated for 48 weeks with PegIFN plus ribavirin

C.-Y. Dai $(\bowtie)$

Hepatobiliary Division, Department of Internal Medicine, Kaohsiung Medical University Hospital, Kaohsiung Medical University, No. 100, Tzyou 1st Road, San-Ming District,

Kaohsiung 807, Taiwan

e-mail: daichiayen@gmail.com

C.-Y. Dai

Department of Occupational and Environmental Medicine, Kaohsiung Medical University Hospital, Kaohsiung Medical University, No. 100, Tzyou 1st Road, San-Ming District, Kaohsiung 807, Taiwan

\section{C.-Y. Dai}

Faculty of Internal Medicine, College of Medicine, Kaohsiung Medical University, No. 100, Tzyou 1st Road, San-Ming District, Kaohsiung 807, Taiwan
1,000-1,200 mg/day and patients with genotype 2 or 3 infection are treated for 24 weeks with PegIFN plus a lower dose of ribavirin at $800 \mathrm{mg} /$ day currently [3, 4]. With the current SOC regimen, the sustained virological response (SVR) rates in Western countries were 42-60\% $[3,5,6]$ and 76-95\% [3, 6, 7] for genotype 1 and 2/3 patients, respectively. The response to the recommended regimens has been observed to be substantially higher in Asian countries [8]. To maximize SVR rates by using the on-treatment viral kinetics [rapid virologic response (RVR): HCV RNA seronegative by PCR $(<50 \mathrm{IU} / \mathrm{ml})$ at week 4 and early virologic response (EVR): HCV RNA seronegative or decline more than two logs from baseline at week 12] is the major advance of therapy for CHC. An RVR is one of the best predictors of an SVR for PegIFN plus ribavirin therapy, irrespective of $\mathrm{HCV}$ genotype [9-11]. Several studies in Western countries have reported that HCV-1/4-infected patients who have an RVR during PegIFN/ribavirin have high SVR rates when the treatment course is shortened to 24 weeks, in particular among patients with lower baseline viral loads (84-93\%). The HCV-2/3-infected patients who have an RVR during PegIFN plus weight-based dose of ribavirin have high rates of SVR (82-90\%) when the treatment course is shortened to $12-16$ weeks.

In Taiwan, truncation of treatment to 16 weeks for genotype 2 patients with a weight-based ribavirin dose for genotype 1 patients achieves $100 \%$ of SVR rate [11] and truncation of treatment to 24 weeks for genotype 1 patients with lower baseline viral loads achieves $94-96 \%$ of SVR rate [9]. The merits of a shortened treatment regimen, which can be attempted without influencing treatment efficacy, include reducing costs of drugs and medical testing as well as improving tolerability for patients, which ensures compliance throughout the course of treatment. 
In this issue of the journal, Yan et al. [12] reported their retrospective study to evaluate the treatment rate of $\mathrm{CHC}$ patients and examine the reasons for non-treatment in a tertiary referral center in Hong Kong. Of their enrolled 303 CHC patients, 138 (45.5\%) patients received no antiviral therapy. The reasons for non-treatment, classified as due to patients' preference $(33.3 \%)$ or according to physicians' decisions $(66.6 \%)$, were as follows: $31.9 \%$ declined treatment, $18.8 \%$ had decompensated cirrhosis, $12.3 \%$ were considered too elderly (range 64-79 years), $17.4 \%$ had too mild liver disease, $7.2 \%$ had psychiatric history, $7.2 \%$ had significant comorbidities, and $2.9 \%$ had ongoing alcohol or substance abuse. The authors concluded that factors including older age, significant comorbidities, psychiatric history, mild liver disease and decompensated cirrhosis were associated with non-treatment. The results reported locally in this study shed light on the general status and current concerns about applying anti-HCV therapy in reallife care settings. Several major hindrances encountered currently have to be overcome and some issues need to be addressed.

Firstly, encouragement of physicians to treat and encourage patients to receive therapy if the result of therapy is excellent is deserving of attention. Since the response to SOC therapy has been improved, the current strategies should increase the rate of therapy. Particularly increasing evidence indicates that Asian patients have a higher likelihood to achieve an SVR than Caucasians. Ge et al. [13] reported that Asian patients had the highest frequency of the advantageous allele in the gene for interleukin 28B, which has been shown to be a significant predictor of response in Asian studies [14, 15]. Hence, it is reasonable that Asian patients should be considered more favorably to receive treatment for CHC. It seems undoubtedly true that 'easier-to-treat' patients (HCV genotypes $2 / 3$ with RVR) have an extremely high SVR rate most suitable for therapy.

Secondly, patient preference not to receive therapy is another important issue. Although PegIFN plus ribavirin is cost-effective for the initial treatment of $\mathrm{CHC}$, it is not uncommon that the cost for anti-HCV therapy will lead to patients rejecting therapy, as $12.3 \%$ of patients were not treated as reported by Yan et al. [12] owing to the strict criteria for full government reimbursed antiviral therapy. In Taiwan, a good example is that the cost of therapy is currently reimbursed by National Health Insurance for 24-48 weeks regimen according to the achieving RVR or not. Under this circumstance, more Taiwanese patients can be expected to receive therapy. Another important consideration is the side-effects caused by PegIFN and ribavirin, which might terrify both patients and physicians when these can be particularly disabling or even life-threatening. The management of side-effects has improved the quality of life and therefore patient adherence to therapy by using medications such as acetaminophen for flu-like symptoms, antidepressants, and anxiolytics for neuropsychiatric disorders and hematopoietic growth factors for neutropenia or anemia. Careful monitoring is necessary and a thorough discussion between physicians and patients regarding benefits to prevent potential liver complications, side-effects and their management, and the effectiveness and prognosis of antiviral treatment is critical.

Lastly, increasing evidence supports the treatment of CHC patients who are not considered eligible for therapy in randomized registration trials or with significant comorbidities. For patients over the age of 65 who respond more poorly than younger patients to conventional interferon alfa (IFN) plus ribavirin, some data have shown a similar SVR rate to younger patients when they are treated with and adhere to PegIFN plus ribavirin therapy $[16,17]$. The important association between type 2 diabetes mellitus (DM) and insulin resistance (IR), measured as homeostasis assessment (HOMA) score, and chronic HCV infection has become noticeable recently. DM may have an etiological role in chronic liver disease and HCC regardless of alcohol and viruses [18] and high serum glucose level and IR are all associated with severity of fibrosis in CHC patients $[19,20]$. Thus, CHC patients with DM or increased level of IR are more likely to need eradication of HCV than others.

The further challenge we have to face clinically is that patients with increasing levels of IR, unfortunately, achieve SVR less frequently when treated with PegIFN plus ribavirin regardless of HCV genotypes [21, 22]. Correcting overweight and IR by a strict low-calorie diet before SOC treatment can significantly improve the metabolic conditions and the SVR rate for CHC patients with metabolic syndrome [23]. Using an insulin-sensitizing agent such as metformin or a peroxisome proliferation-acting receptor -gamma agonist, such as rosiglitazone or pioglitazone might be an alternative approach to overcome the suboptimal antiviral response to SOC therapy in patients with IR [24].

In a study by Yan et al. [12], concurrent infection of $\mathrm{HBV}$ and $\mathrm{HCV}$ in $5.6 \%$ of $\mathrm{CHC}$ patients is more common where both viruses are endemic [25, 26] and no current treatment is approved for this important patient group yet. An open-label, comparative, multicenter study conducted in Taiwan has shown recently that PegIFN 2a plus ribavirin is equally effective in patients with $\mathrm{HCV}$ monoinfection (SVR: $77 \%$ in genotype 1 and $84 \%$ in genotype 2/3) and in those with dual chronic HCV/HBV infection and negative for hepatitis B e antigen (HBeAg) (SVR: 72\% in genotype 1 and $83 \%$ in genotype $2 / 3$ ). Besides, reactivation of HBV DNA at 6 months after treatment was observed in $36.3 \%$ of HBV DNA-negative patients [27]. The results dramatically suggest that SOC regimen in $\mathrm{HCV}$ monoinfected patients 
can be recommended for this patient group. However, the long-term safety profile in these patients treated with SOC regimen and whether the treatment can be effective for patients with dual chronic $\mathrm{HCV} / \mathrm{HBV}$ infection and positive $\mathrm{HBeAg}$ need to be clarified in the future.

Hemodialysis (HD) patients are in great risk of $\mathrm{HCV}$ infection. Anti-HCV treatment for HD patients is important because $\mathrm{HCV}$ infection increases mortality in HD patients [28]. The SOC therapy has not been studied thoroughly in HD patients because of the concerns about the side-effects of the medication, particularly the hemolytic anemia induced by ribavirin. Two studies with SOC therapy showed SVR rates of 97\% [29] and 44\% [30]. It is not surprising that the median ribavirin dosage was $800 \mathrm{mg} /$ week (range, 600-1,000 mg/week) in HD studies, which reminds physicians to closely and carefully monitor the hemoglobin level and adjust the ribavirin dose accordingly during SOC therapy.

Even the efficacy of SOC therapy has markedly improved, and further efforts are mandatory to help physicians and $\mathrm{CHC}$ patients enjoy more effective, comfortable, and safer treatment. We have to explore more predictors, including pre-treatment, on-treatment, viral or host, for responses which will lead us to design more optimal regimens in individualized therapy. A truncated regimen or reduced dose of medication without decreasing the SVR rate seems to directly enhance the willingness of patients to receive therapy. More studies to extend and confirm the applicable indications for the current SOC regimen to advantage more $\mathrm{CHC}$ patients to eradicate $\mathrm{HCV}$ are necessary. For example, guidelines for the treatment of $\mathrm{HCV}$ infection stated that treatment of HCV infection should not be withheld from IV drug users provided they wish to take HCV treatment, are able to maintain close monitoring, and practice contraception [4].

Recently, a prospective, case-control study conducted in Taiwan has shown that in patients with achievement of good adherence in terms of 80/80/80 adherence (on $>80 \%$ of their IFN and ribavirin dosage for the duration of treatment) for SOC, the efficacies were comparable regardless of the $\mathrm{HCV}$ genotype between $\mathrm{CHC}$ patients with $\mathrm{HCC}$ who have received curative treatment and those with cirrhosis [31]. For children with CHC, the US Food and Drug Administration (FDA) has recently approved combination of PegIFN-2b and RBV for use in previously untreated children with chronic hepatitis $\mathrm{C}$ aged 3 years or older due to the favorable results [32]. Furthermore, recent progress in the development of novel medications including albinterferon, more longer acting recombinant IFN, and direct-acting antivirals (DAAs), which might enhance SVR rates and diminish length of treatment when combined with current SOC regimen will lead us to a new era of $\mathrm{HCV}$ treatment.

\section{References}

1. Chuang WL, Chang WY, et al. The role of hepatitis B and C viruses in hepatocellular carcinoma in a hepatitis B endemic area: a case-control study. Cancer. 1992;69:2052-2054.

2. Fattovich G, Stroffolini T, Zagni I, Donato F. Hepatocellular carcinoma in cirrhosis: incidence and risk factors. Gastroenterology. 2004;127:S35-S50.

3. Hadziyannis SJ, Sette H Jr, Morgan TR, et al. Peginterferonalpha2a and ribavirin combination therapy in chronic hepatitis $\mathrm{C}$ : a randomized study of treatment duration and ribavirin dose. Ann Intern Med. 2004;140:346-355.

4. Strader DB, Wright T, Thomas DL, Seeff LB. Diagnosis, management, and treatment of hepatitis C. Hepatology. 2004;39:1147-1171.

5. Manns MP, McHutchison JG, Gordon SC, et al. Peginterferon alfa- $2 b$ plus ribavirin compared with interferon alfa- $2 b$ plus ribavirin for initial treatment of chronic hepatitis $\mathrm{C}$ : a randomized trial. Lancet. 2001;358:958-965.

6. Zeuzem S, Pawlotsky JM, Lukasiewicz E, et al. International, multicenter, randomized, controlled study comparing dynamically individualized versus standard treatment in patients with chronic hepatitis C. J Hepatol. 2005;43:250-257.

7. Mangia A, Santoro R, Minerva N, et al. Peginterferon alfa- $2 \mathrm{~b}$ and ribavirin for 12 vs. 24 weeks in HCV genotype 2 or 3. $N$ Engl J Med. 2005;352:2609-2617.

8. Yu ML, Chuang WL. Treatment of chronic hepatitis C in Asia: when East meets West. J Gastroenterol Hepatol. 2009;24:336345.

9. Yu ML, Dai CY, Huang JF, et al. Rapid virological response and treatment duration for chronic hepatitis $\mathrm{C}$ genotype 1 patients: a randomized trial. Hepatology. 2008;47:1884-1893.

10. Liu CH, Liu CJ, Lin CL, et al. Pegylated interferon-alpha-2a plus ribavirin for treatment-naive Asian patients with hepatitis $\mathrm{C}$ virus genotype 1 infection: a multicenter, randomized controlled trial. Clin Infect Dis. 2008;47:1260-1269.

11. Yu ML, Dai CY, Huang JF, et al. A randomised study of peginterferon and ribavirin for 16 versus 24 weeks in patients with genotype 2 chronic hepatitis C. Gut. 2007;56:553-559.

12. Yan KK-L, Wong GL-H, Wong VW-S, Chan HL-Y. Rate and factors affecting treatment uptake of patients with chronic hepatitis C in a tertiary referral hospital. Dig Dis Sci. 2010. doi: 10.1007/s10620-010-1412-1.

13. Ge D, Fellay J, Thompson AJ, et al. Genetic variation in IL28B predicts hepatitis C treatment-induced viral clearance. Nature. 2009;461:399-401.

14. Tanaka Y, Nishida N, Sugiyama M, et al. Genome-wide association of IL28B with response to pegylated interferon-alpha and ribavirin therapy for chronic hepatitis C. Nat Genet. 2009;41: 1105-1109.

15. Yu ML, Huang CF, Huang JF, et al. Role of interleukin-28B polymorphisms in the treatment of hepatitis $\mathrm{C}$ virus genotype 2 infection in Asian patients. Hepatology. 2010 (in press), doi: 10.1002/hep.23976.

16. Huang CF, Yang JF, Dai CY, et al. Efficacy and safety of pegylated interferon combined with ribavirin for the treatment of older patients with chronic hepatitis C. J Infect Dis. 2010;201: 751-759.

17. Antonucci G, Longo MA, Angeletti C, et al. The effect of age on response to therapy with peginterferon alpha plus ribavirin in a cohort of patients with chronic HCV hepatitis including subjects older than 65 year. Am J Gastroenterol. 2007;102: $1383-1391$.

18. El-Serag HB, Everhart JE. Diabetes increases the risk of acute hepatic failure. Gastroenterology. 2002;122:1822-1828. 
19. Ratziu V, Munteanu M, Charlotte F, Bonyhay L, Poynard T. Fibrogenic impact of high serum glucose in chronic hepatitis C. J Hepatol. 2003;39:1049-1055.

20. Hui J, Sud A, Farrell GC, et al. Insulin resistance is associated with chronic hepatitis $\mathrm{C}$ virus infection and fibrosis progression. Gastroenterology. 2003;125:1695-1704.

21. Poustchi H, Negro F, Hui J, et al. Insulin resistance and response to therapy in patients infected with chronic hepatitis $\mathrm{C}$ virus genotypes 2 and 3. J Hepatol. 2008;48:28-34.

22. Dai CY, Huang JF, Hsieh MY, et al. Insulin resistance predicts response to peginterferon-alpha/ribavirin combination therapy in chronic hepatitis C patients. J Hepatol. 2009;50:712-718.

23. Tarantino G, Conca P, Ariello M, Mastroliae M. Does a lower insulin resistance affect antiviral therapy response in patients suffering from HCV related chronic hepatitis? Gut. 2006;55:585.

24. Romero-Gómez M, Diago M, Andrade RJ, et al. Treatment of insulin resistance with Metformin in naïve genotype 1 chronic hepatitis $\mathrm{C}$ patients receiving peginterferon alfa-2a plus ribarivin. Hepatology. 2009;50:1702-1708.

25. Liu CJ, Liou JM, Chen DS, et al. Natural course and treatment of dual hepatitis B virus and hepatitis C virus infections. J Formos Med Assoc. 2005;104:783-791.

26. Dai CY, Ho CK, Huang JF, et al. Hepatitis $\mathrm{C}$ virus viremia and low platelet count: a study in a hepatitis B \& $\mathrm{C}$ endemic area in Taiwan. J Hepatol. 2010;52:160-166.
27. Liu CJ, Chuang WL, Lee CM, et al. Peginterferon Alfa-2a plus ribavirin for the treatment of dual chronic infection with hepatitis B and C viruses. Gastroenterology. 2009;136:496-504.

28. Fabrizi F, Martin P, Dixit V, Bunnapradist S. Dulai G: Metaanalysis: Effect of hepatitis $\mathrm{C}$ virus infection on mortality in dialysis. Aliment Pharmacol Ther. 2004;20:1271-1277.

29. Rendina M, Schena A, Castellaneta NM, et al. The treatment of chronic hepatitis $\mathrm{C}$ with peginterferon alfa-2a $(40 \mathrm{kDa})$ plus ribavirin in haemodialysed patients awaiting renal transplant. J Hepatol. 2007;46:768-774.

30. Deltenre V, Canva F, Provot F, et al. Pegylated interferon and ribavirin in haemodialyzed patients with chronic hepatitis $\mathrm{C}$ : A prospective study. Hepatology, 2006; 44: S329A.

31. Huang JF, Yu ML, Huang CF, et al. The efficacy and safety of pegylated interferon plus ribavirin combination therapy in chronic hepatitis $\mathrm{C}$ patients with hepatocellular carcinoma post curative therapies - a multicenter prospective trial. J Hepatol. 2010 (in press).

32. Wirth S, Ribes-Koninckx C, Calzado MA, et al. High sustained virologic response rates in children with chronic hepatitis $\mathrm{C}$ receiving peginterferon alfa- $2 \mathrm{~b}$ plus ribavirin. $\mathrm{J}$ Hepatol. 2010;52:501-507. 